\title{
The 10-year bioanalysis challenge in Mexico
}

\author{
Gabriel Marcelín-Jiménez*,1 \\ ${ }^{1}$ Pharmometrica. Av. de las Granjas 972, Edif. "C" planta baja - Tecnoparque Azcapotzalco, Col. Santa Bárbara, 02230 Ciudad de \\ México $(C D M X)$, Mexico \\ *Author for correspondence: Tel.: +52 559127 0111; gabmarcelin@pharmometrica.com.mx
}

\section{'6 BE studies have depicted pharmacokinetics of several drugs in our population, that in some cases have contributed to modify the information for prescription according to our genetic background and phenotype."}

First draft submitted: 31 January 2019; Accepted for publication: 21 February 2019; Published online: 17 April 2019

Keywords: bioanalysis • México

Bioanalysis is the 'artistic' aspect of analytical chemistry related to the task of extracting analytes from biological matrices or tissues for identification and/or quantitation, which is my personal opinion formed over the last two decades working in PK and drug monitoring. The chemical structure of analytes is also of course important, as it will drive the efforts and strategy for their selective, and in some cases specific, extraction. There is a huge variety of analytes and modalities explored by bioanalysis today, for example, carbohydrates, lipid derivates, nucleic acids, proteins and, in the case of my field of experience, small-molecule drugs and their metabolites that once separated from the biological matrix, are introduced into instrumental platforms for isolation, characterization and quantification.

Most such laboratory work are carried out in Mexico under a regulatory framework, including bioequivalence and PK trials, food and safety analysis and forensic studies. Other work is performed in a semi-regulated manner, such as the search for pollutants in water and soil, biotech and clinical biomarkers research or basic life sciences.

The aim of this editorial is to share a brief and personal description of how bioanalysis has evolved in Mexico in certain areas of application over the last 10 years. For ease of reading of the article, I have divided bioanalysis into three stages: extraction, instrumentation (chromatography and mass spectrometry) and regulatory affairs.

\section{Extractive procedures}

The amount of biological material used for extracting analytes has diminished drastically with the arrival of more sensitive detection systems over the years, evolving from the use of amounts in grams of tissue to milligrams of samples; and in the case of liquid samples, from milliliters of blood or serum to a few microliters. Basic strategies for extract analytes remain constant (disruption of solid tissues by grinding or ultrasonic baths), protein precipitation, LLE, ultra-filtration and SPE; however, the scale at which it is implemented has been minimized. The adoption of the 96-microwell plates format for SPE and even for protein precipitation, and phospholipids elimination results in semi-automated high-throughput laboratory routines, which produce accurate and robust results in a reduced amount of time (fully automated extractive systems are not common in Mexico due to the high cost of such platforms).

In Mexico, the use of organic chlorinated solvents (e.g., methylene chloride, dichloromethane and chloroform) have been forbidden over the last decade, and the development of micro-LLE procedures provide less quantity of evaporated solvents that results in ecological sample preparation techniques.

One sample technique that had high expectations and promise was the use of 'dried blood spots (DBS)'; several papers were published for its use in PK and preclinical studies [1]. Unfortunately, DBS was not fully accepted by the Mexican regulatory authorities mainly because no one bioequivalence study was submitted in any other international regulatory agency based on this collecting, transporting and storage technique. Spotting of liquid samples in monolayers was difficult to standardize, influence of hematocrit has been always controversial, there was not agreement concerning the addition of internal standard over the paper cards or during extraction of spots, 
and finally quantitation of drugs at very low level (nanograms or even picograms) was not precise and accurate enough $[2,3]$.

Although these, DBS with MS spring in preclinical toxicology and pharmacology, and for clinical investigation of novel therapeutic treatments in pediatrics [4].

\section{Chromatography \& MS}

The last decade was the witness of the obsolescence of HPLC with ultra-violet (UV-VIS) detection for bioanalysis. The sub-1.8- $\mu \mathrm{m}$ particle chromatographic columns consolidates the UHPLC as the gold standard of separation techniques. This micro-fluidic technique allowed the coupling with different kind of mass spectrometers, such as quadrupole-traps $(\mathrm{Qt})$, time-of-flight $(\mathrm{ToF})$ and the most popular tandem mass spectrometers (MS/MS); thus, resulting in many cases in hybrid platforms with several troubles of software intercommunication. Maturation of our regulation avoid such kind of configurations, allowing only platforms of the same vendor and with a unique controlling software. UHPLC coupled with MS/MS becomes the golden standard for quantitation of small drugs in Mexico.

Concerning to more advances in chromatography, supercritical fluids chromatography arrived, improving separation of very complex mixtures of vitamins, steroids and racemic drugs, and also used at preparative level of plant and animal extracts (that may be considered a type of bioanalysis).

In the case of spectrometers, tandem mass spectrometers arrived as universal detectors, substituting other types of detectors such as fluorometers, coulometers, diffractometers and even spectrophotometers UV-VIS, by offering several orders of magnitude in sensitivity and extreme selectivity, with the unique condition that molecules could be ionizable. Every time a new generation of spectrometers comes to the scene, they improve in sensitivity, offering the perfect tools for the advance of lipidomics and metabolomics.

Another electromagnetic instrument that arrived coupled with the next generation of ToF spectrometers were the ion mobility devices, high vacuum linear tubes that with the use of high voltages and magnetic pulses accelerate biomolecules (mainly proteins), separating them based on the velocity that molecules acquire due to their shape, providing information not only of mass, but also of tridimensional conformation of such biopolymers, contributing with the knowledge of genetics, proteomics and biotech drug analysis.

\section{Regulatory affairs}

The Health and the Agriculture Ministries were strengthened with the equipment of their own laboratories for their regulatory efforts [5]. Mexican Pharmacopeia was one of the first pharmacopeias to include MS in the 'General Methods' chapter, and proposed a list of 'Good chromatographic practices' for bioanalysis [6]. Finally, Mexican regulatory guidelines for bioequivalences has been updated twice, including validation criteria for bioanalytical methods focused in the quantitation of drugs - small molecules - in biological fluids based on MS [7].

In Mexico, during the last 10 years, the field of regulated bioanalysis that grew up exponentially was bioequivalence (BE), having from tens of authorized protocols at the beginning to more than 500 trials authorized by COFEPRIS (Mexican Regulatory Agency) last year, and performed in 'third-part authorized research centers' - a legal figure that only exists in Mexico - [8]. BE studies have depicted pharmacokinetics of several drugs in our population, that in some cases have contributed to modify the information for prescription according to our genetic background and phenotype. $\mathrm{BE}$ and generic drugs have strengthened the national pharmaceutic industry, and give our population medicines as safe and effective as patented drugs at low cost.

Concluding, bioanalysis is a thrilling activity in constant evolution that pushes the researchers to know more about chemistry, biochemistry, instrumentation, physic-chemistry, statistics and pharmacology. Mexican investigators have participated in global forums for harmonization on bioanalysis of small molecules, and contribute continuously with the enrichment in the field of plant genomics, analysis of biotech products and pharmacokinetics [9-11]. It remains many things to do, particularly in making our regulatory agency more efficient, and in coupling the efforts done by Universities with the needs of the local industry.

Financial \& competing interests disclosure

The author has no relevant affiliations or financial involvement with any organization or entity with a financial interest in or financial conflict with the subject matter or materials discussed in the manuscript. This includes employment, consultancies, honoraria, stock ownership or options, expert testimony, grants or patents received or pending, or royalties.

No writing assistance was utilized in the production of this manuscript. 


\section{References}

1. González A, Contreras L, Angeles A et al. Pharmacokinetics of diphenylboroxazolidones of 1- $\alpha$-amino acids with activity on the CNS: quantification in rat DBS by UPLC-MS/MS. Bioanalysis 3(4), 439-448 (2011).

2. Li W, Tse FL. Dried blood spot sampling in combination with LC-MS/MS for quantitative analysis of small molecules. Biomed. Chromatogr. 24(1), 49-65 (2010).

3. Timmerman P, White S, Cobb Z et al. Update of the EBF recommendation for the use of DBS in the regulated bioanalysis integrating the conclusions from the EBF DBS Microsampling Consortium. Bioanalysis 5(17), 2129-2136 (2013).

4. Contreras L, Rivera L, Moreno A et al. Development of a UPLC-MS/MS method for the quantification of sildenafil by DBS, and its use on pediatric pulmonary hypertension. Bioanalysis 6(21), 2815-2824 (2014).

5. Norma Oficial Mexicana NOM-004- ZOO-1999. Control of toxic residues in meat, fat, liver and kidney of bovines, equines, porcines and ovines. SAGARPA, Mexico (1999). http://www.porcimex.org/NORMAS/NOM-004-ZOO-1994.pdf

6. Mexican Pharmacopeia. Farmacopea de los Estados Unidos Mexicanos. Secretaría de Salud. Métodos Generales de Análisis (2019). https://www.farmacopea.org.mx/

7. Norma Oficial Mexicana NOM-177-SSA1-2013. Guidelines to establish bioequivalence between test and reference formulations. Guidelines to establish biocomparability between biotech products. http://www.dof.gob.mx/nota_detalle.php?codigo=5314833\&fecha=20/09/2013

8. National Register of Clinical Assays in Mexico (RNEC-COFEPRIS). http://siipris03.cofepris.gob.mx/Resoluciones/Consultas/ConWebRegEnsayosClinicos.asp

9. Woolf E, McDougall S, Fast D et al. Small molecule specific run acceptance, specific assay operation, and chromatographic run quality assessment: recommendation for best practices and harmonization from the Global Bioanalysis Consortium harmonization teams. AAPS J. 16(5), 885-892 (2014).

10. Marcelín-Jiménez G. How pitfalls during drug quantitation by mass spectrometry may affect the variability of pharmacokinetic data during a bioequivalence trial. J. Bioequiv. Availab. 9(4), 430-431, (2017).

11. Peñaloza C, Marcelín JG, López M et al. Development of memantine orodispersible tablets and their in vitro description and biopharmaceutical performance. Int. J. Pharmacokinet. 3(1), 13-21, (2018). 
